\title{
Could the Platelet-to-Lymphocyte Ratio be a Novel Marker for Predicting Invasiveness of Cervical Pathologies?
}

\author{
Mesut Kose, Fatih Celik*, Seda Kayman Kose, Dagistan Tolga Arioz, Mehmet \\ Yilmazer
}

\begin{abstract}
Purpose: To determine whether the preoperative platelet to lymphocyte ratio (PLR) could predict invasiveness of cervical pathologies. Materials and Methods: Patients with preinvasive and invasive diseases were reviewed retrospectively, over a nine-year period, 2005-2014. The pathological records and completed blood counts of the patients were collected and recorded in the SPSS program. Patients were divided in two groups, preinvasive and invasive. Results: The median PLR was significantly higher in the invasive group than in the preinvasive group $(p=0.03)$. There was a correlation between invasion of cervical cancer and white blood cell count, red cell distributing width (RDW), neutrophil-lymphocyte ratio (NLR), and PLR. Conclusions: This study showed that patients with uterine cervical cancer may present with leukocytosis, increased RDW, NLR and PLR. These cheap and easily available parameters, especially PLR, may provide useful information about the invasiveness of cervical lesions.
\end{abstract}

Keywords: Platelet to lymphocyte ratio - cervical pathologies - invasive - pre-invasive

Asian Pac J Cancer Prev, 16 (3), 923-926

\section{Introduction}

The term preinvasive disease of the cervix (PCD) was first defined in 1947 (Pund, 1947). Despite the fact that epithelial changes give the appearance of invasive cancer on the cervix, lesions confined to the epithelium are to be noted in PCD. After PCD is defined, some studies have shown that if these lesions did not treat cervical dysplasia, it progresses to cervical cancer (Koss et al., 1963). Therefore, when the preinvasive lesions could be diagnosed before the stage of the invasive cervical cancer, this early detection allows easy treatment (Richart \& Sciarra, 1968).

Cervical cancer (CC) is still one of the most frequent malignancies in women worldwide (Siegel, Naishadham \& Jemal, 2013). In CC, like the other cancers, the host response to malignant tumors comprises not only changes in the microenvironment, but also systemic alterations (Tavares-Murta et al., 2010). One of the important systemic alterations is inflammation. Inflammation is a nonspecific feature of cancer and plays an important role in various aspects of cancer involving cancer initiation, promotion, progression, metastasis and clinical features (Babu et al., 2012). Therefore, inflammatory markers were studied in various cancer types as indicators of invasion (Acmaz et al., 2014).

The platelet-lymphocyte ratio (PLR) is an inflammatory marker and has been studied in uterine cancer and uterine cancer precursor lesions (Acmaz et al., 2014). The aim of the present study is to evaluate the relationship between PLR and cervical cancer invasiveness.

\section{Materials and Methods}

\section{Patients}

Patients of the Afyon Kocatepe University Obstetrics and Gynecology Service with preinvasive and invasive diseases were reviewed retrospectively, over a nine-year period, 2005-2014. The data of the cases were collected from hospital records and patients files. Firstly, the pathological records of the patients were examined and the results were recorded to SPSS. Secondly, the patient's completed blood counts were recorded in the SPSS program, and were recorded on the date of pathologic diagnosis prior to any treatment. Patients were excluded if they had received previous cervical neoplasia treatment.

Patients were divided in two groups, preinvasive and the second was the invasive group. All were evaluated according to standard complete blood count (CBC) results. The white blood cells (WBC), red cell distributing width (RDW), absolute neutrophil count, absolute lymphocyte count, and absolute platelet count were employed as parameters of interest. The neutrophil-lymphocyte ratio (NLR) was defined as the absolute neutrophil count divided by the absolute lymphocyte count; similarly, the platelet-lymphocyte ratio (PLR) was defined as the absolute platelet count divided by the absolute lymphocyte count. 


\section{Statistical analysis}

SPSS 20 statistics software was used for the statistical analysis. The Kolmogorov-Smirnov test was used to determine the distribution of the variables. The median value (min-max) was used in variables without normal distribution. The Mann-Whitney U-test was used for continuous variables without normal distribution. Pearson's correlation analysis was used to determine the correlation between invasion of cervical cancer and WBC, RDW, NLR, and PLR. The ROC curve analysis was used to assess the discriminative role of $\mathrm{WBC}, \mathrm{RDW}, \mathrm{NLR}$, and PLR levels. A p value of $<0.05$ value was accepted as significant.

\section{Results}

Table 1 shows a comparison of age and blood parameters among the two groups. The mean age was 41.6 \pm 9.7 years in patients with preinvasive pathology (Group 1) and the mean age was $54.3+10.8$ years old in patients with cervical cancer (Group 2). The median WBC was statistically significantly higher in Group 2 than Group $1(\mathrm{p}=0.001)$. The median WBC was $7.3 \pm 1.7$ in Group 1 and 9.4+4.3 in Group 2. The median RDW was $14.5 \pm 2.8$ in Group 1 and $15.8 \pm 2.5$ in Group 2. There was a statistically significant difference between the groups in terms of RDW ( $\mathrm{p}=0.006)$. The median neutrophil to lymphocyte ratio was $2.1 \pm 0.9$ in Group 1 and $13.8 \pm 59.3$ in Group 2. There were statistically significant differences between the groups according to NLR ( $\mathrm{p}=0.003$ ) (Figure 1). The median PLR was statistically significantly higher in Group 2 than in Group 1 ( $\mathrm{p}=0.03$ ) (Figure 2).

There was a correlation between invasion of cervical cancer and WBC, RDW, NLR, and PLR as shown in Table 2. However, while the correlation between invasion of cervical cancer and WBC, RDW, PLR was statistically

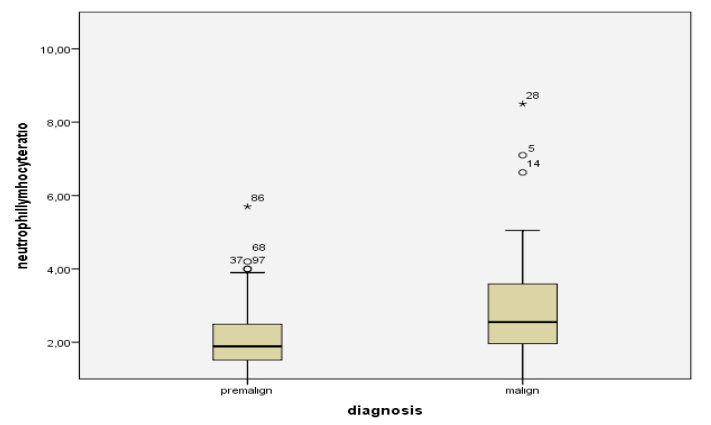

Figure 1. Mean NLR Values of Patients with Cervical Preinvasive Disease And Cervical Cancer. NLR: neutrophil-to-lymphocyte ratio $(\mathrm{p}=0.003)$ significant, it was not statistically significant between NLR and invasion of the cervical cancer.

The ROC curve analysis (Figure 1) demonstrated that WBC, RDW, NLR, and PLR may be discriminative parameters for invasion of cervical cancer. The area under the curve (AUC), cut off values, and sensitivity and specify of ROC are depicted in Table 3. The area under the curve values for WBC, RDW, NLR, and PLR were 0.708, 0.673, 0.687 , and 0.630 , respectively.

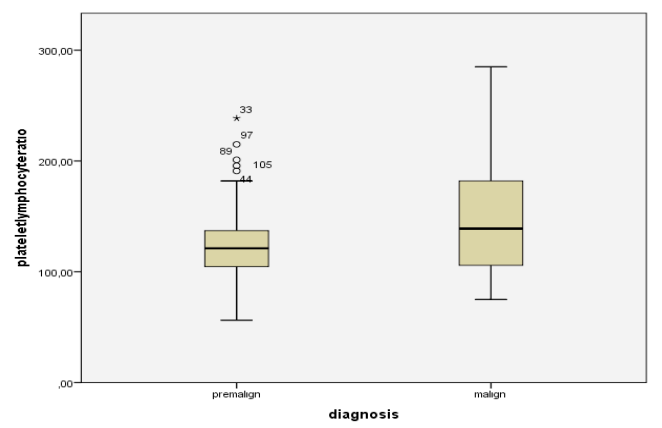

Figure 2. Mean PLR Values of the Patients with Cervical Preinvasive Disease and Cervical Cancer. PLR: platelet-to-lymphocyte ratio $(\mathrm{p}=0.03)$

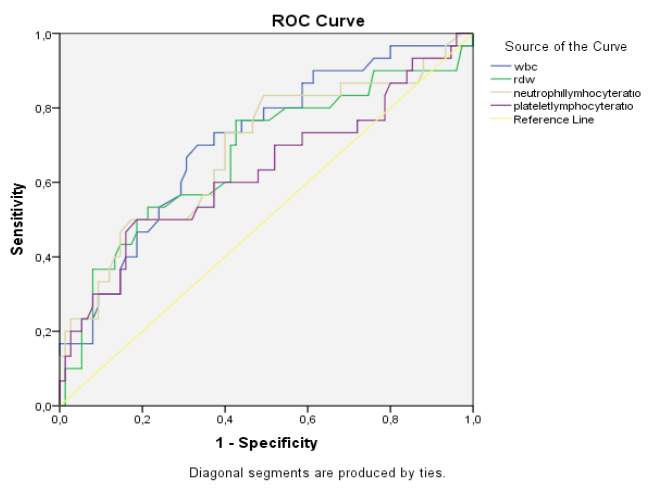

Figure 3. ROC Curve Demonstrating the AUC of WBC,RDW,NLR and PLR for Invasion of Cervical Pathologies

Table 2. Correlation between Invasion of Cervical Pathologies and Inflammatory Markers

\begin{tabular}{|c|c|c|c|}
\hline & WBC & RDW & PLR \\
\hline $\begin{array}{l}\text { Invasion of cervical } \\
\text { pathologies }\end{array}$ & $\begin{aligned} \mathrm{CC} & =0.336 \\
\mathrm{p} & =0.00\end{aligned}$ & $\begin{aligned} \mathrm{CC} & =0.2 \quad \mathrm{CC}=0.16 \\
\mathrm{p} & =0.04 \quad \mathrm{p}=0.87\end{aligned}$ & $\begin{aligned} \mathrm{C} & =0.275 \\
\mathrm{p} & =0.005\end{aligned}$ \\
\hline
\end{tabular}

Table 1. Comparison of Age and Blood Parameters among the Two Groups

\begin{tabular}{lccc}
\hline & Patients with preinvasive pathology $(\mathrm{n}=75)$ & Patients with cancer $(\mathrm{n}=30)$ & $\mathrm{P}$ value \\
\hline Age (year) & $41.6 \pm 9.7$ & $54.3 \pm 10.8$ & 0 \\
White blood cell count $(/ \mathrm{mL})$ & $7.3 \pm 1.7$ & $9.4 \pm 4.3$ & 0.001 \\
Red cell distribution widty $(\%)$ & $14.5 \pm 2.8$ & $15.8 \pm 2.5$ & 0.006 \\
Neutrophil to lymphocyte ratio & $2.1 \pm 0.9$ & $13.8 \pm 59.3$ & 0.003 \\
Platelet to lymphocyte ratio & $125.2 \pm 33.3$ & $150.2 \pm 53.2$ & 0.03 \\
\hline
\end{tabular}

*All of the inflammatory parameters and age were significantly higher in the group of patients with cancer compared to patients in the group with preinvasive cervical pathology 
Can the Platelet-to-Lymphocyte Ratio be a Novel Marker for Predicting Invasiveness of Cervical Pathologies?

Table 3. Area Under Curve(AUC), Standard Cut-off Values and Senstivity and Specifity for WBC, RDW, NLR and PLR in Cervical Cancer Patients.

\begin{tabular}{lllllc}
\hline & AUC & Std Error & 95\%CI & $\begin{array}{c}\text { Cut off } \\
\text { value }\end{array}$ & $\begin{array}{c}\text { Sensitivity\%- } \\
\text { Specivity\% }\end{array}$ \\
\hline WBC $(/ \mathrm{mL})$ & 0.708 & 0.056 & 0.598 & 8050 & $73.3-63.7$ \\
RDW $(\%)$ & 0.673 & 0.062 & 0.551 & 14 & $76.7-58.3$ \\
NLR & 0.687 & 0.61 & 0.568 & 2.01 & $73-54$ \\
PLR & 0.63 & 0.65 & 0.502 & 111000 & $73.3-54.7$ \\
\hline
\end{tabular}

*WBC:White blood cell count, RDW: Red cell distridution widty, NLR: Neotrophil to lymphocyte ratio, PLR: Platelet lymphocyte ratio. CC: correlation coefficient

\section{Discussion}

According to our knowledge, this is the first study investigating the relationship between PLR, RDW, and cervical preinvasive and invasive disease. It was found that RDW and PLR were associated with invasive cervical disease.

Leukocyte migration is at the center of the inflammation as a response to tumor formation. Leukocytosis has been evaluated in many studies in lung cancers and colorectal cancer (Sprague et al., 2008; Walsh et al., 2005). The authors have demonstrated that leukocytosis is a risk and prognostic factor in both cancers. The association between leukocytosis and the stage of cervical cancer was demonstrated in another study (Tavares-Murta et al., 2010). The authors concluded that leukocytosis is closely associated with tumor stage. Similarly, leukocyte count was significantly higher in the cervical cancer group in the current study.

RDW is a component of the standard complete blood count (CBC) and generally used for the differential diagnosis of microcytic anemias (Bessman, 1985). RDW has been studied as an inflammatory marker in many chronic inflammatory diseases such as colon cancer, inflammatory bowel disease, arthritis, and Alzheimer's disease (Spell et al., 2004; Lee and Kim, 2010; Yesil et al., 2011; Ozturk et al., 2013). The authors demonstrated that RDW values were significantly elevated in patients with these pathologic conditions. RDW was significantly higher in cervical cancer in the present study, as well.

NLR was defined as a potential marker to determine inflammation in systemic disease (Walsh et al., 2005; Rudiger et al., 2006; Bucak et al., 2013; Ulu et al., 2013; Acmaz et al., 2014). Chronic inflammation in DM, like other chronic diseases, is well known (Schmidt MI, 1999). In a recent study, NLR was reported as a prognostic and predictive marker in diabetic patients with sensorineural hearing loss (Ulu et al., 2013). However, it is well known that inflammation plays an important role in various aspects of cancer. A correlation between the increase of NLR and endometrial cancer invasiveness has been demonstrated (Acmaz et al., 2014). There was an association between NLR and cervical cancer invasiveness in another study (Tavares-Murta et al., 2010). Similarly, in the current study, NLR was higher in the cervical cancer group compared to the preinvasive group. However, there was a statistically significant correlation.

The platelet count is an additional marker of systemic inflammation which is precipitated by the tumor formation. Proinflammatory cytokines such as IL-1 and IL-6 leads to megakaryocyte proliferation and thrombocytosis (Klinger and Jelkmann, 2002; Alexandrakis et al., 2003). Recently, thrombocytosis and lymphocytopenia have been reported as a marker in host systemic inflammation. In the same study, it was emphasized that PLR is a novel marker for inflammation, which incorporates both hematological factors (Smith et al., 2008). In addition, an association between PLR and uterine malign lesions was demonstrated (Acmaz et al., 2014). Furthermore, there was an association and correlation between cervical malign lesions and PLR in the present study. Because of this, the researchers believe that PLR may be a suitable marker like NLR. The main drawback to the current study was its retrospective nature and small sample size.

In conclusion, this study showed that patients with uterine cervical cancer may present with leukocytosis, increased red cell distribution width, neutrophil to lymphocyte ratio and platelet to lymphocyte ratio. These cheap and easily available parameters, especially PLR, may provide useful information about the invasiveness of the cervical pathologies.

\section{References}

Acmaz G, Aksoy H, Unal D, et al (2014). Are neutrophil/ lymphocyte and platelet/lymphocyte ratios associated with endometrial precancerous and cancerous lesions in patients with abnormal uterine bleeding? Asian Pac J Cancer Prev, 15, 1689-92.

Alexandrakis MG, Passam FH, Moschandrea IA, et al (2003). Levels of serum cytokines and acute phase proteins in patients with essential and cancer-related thrombocytosis. Am J Clin Oncol, 26, 135-40.

Babu SN, Chetal G, Kumar S (2012). Macrophage migration inhibitory factor: a potential marker for cancer diagnosis and therapy. Asian Pac J Cancer Prev, 13, 1737-44.

Bessman JD (1985). More on the RDW. Am J Clin Pathology, 84, 773.

Bucak A, Ulu S, Oruc S, et al (2013). Neutrophil-to-lymphocyte ratio as a novel-potential marker for predicting prognosis of bell palsy. Laryngoscope, 124, 1678-81.

Klinger, MH, Jelkmann W (2002). Role of blood platelets in infection and inflammation. J Interferon Cytokine Res, $\mathbf{2 2}$, 913-22.

Koss LG, Stewart F, Foote FW, et al (1963). Some histological aspects of behavior of epidermoid carcinoma in situ and related lesions of the uterine cervix. a long-term prospective study. Cancer, 16, 1160-211.

Lee WS, Kim TY (2010). Relation between red blood cell distribution width and inflammatory biomarkers in rheumatoid arthritis. Arch Pathol Lab Med, 134, 505-6.

Ozturk Z A, Unal A, Yigiter R, et al (2013). Is increased red cell distribution width (RDW) indicating the inflammation in Alzheimer's disease (AD)? Arch Gerontol Geriatr, 56, 50-4.

Pund ER, Nieburgs HE (1947). Preinvasive carcinoma of the cervix uteri: seven cases in which it was detected by examination of rutine endocervical smears. Arch Pathol Lab Med, 44, 571-7.

Richart RM, Sciarra JJ (1968). Treatment of cervical dysplasia by outpatient electrocauterization. Am J Obstet Gynecol, 101, 200-5.

Rudiger A, Burckhardt OA, Harpes P, Muller SA, Follath F (2006). The relative lymphocyte count on hospital admission 


\section{Mesut Kose et al}

is a risk factor for long-term mortality in patients with acute heart failure. Am J Emerg Med, 24, 451-4.

Schmidt MI, Duncan BB, Sharrett AR, (1999). Markers of inflammation and prediction of diabete mellitus in adults:a cohort study. Lancet, 353, 1649-52.

Siegel R, Naishadham D, Jemal A (2013). Cancer statistics, 2013. CA Cancer J Clin, 63, 11-30.

Smith RA, Bosonnet L, Ghaneh P, et al (2008). The plateletlymphocyte ratio improves the the predictive value of serum CA 19.9 levels in determining patient selection for staging laparascopy in suspected periampullary cancer. Surgery, 143, 658-66.

Spell DW, Jones DV, Jr, Harper WF, David Bessman J (2004). The value of a complete blood count in predicting cancer of the colon. Cancer Detect Prev, 28, 37-42.

Sprague BL, Trentham-Dietz A, Klein BE, et al (2008). Physical activity, white blood cell count, and lung cancer risk in a prospective cohort study. Cancer Epidemiol Biomarkers Prev, 17, 2714-22.

Tavares-Murta BM, Mendonca MA, Duarte NL, et al (2010). Systemic leukocyte alterations are associated with invasive uterine cervical cancer. Int J Gynecol Cancer, 20, 1154-9.

Ulu S, Bucak A, Ulu MS, et al (2013). Neutrophil-lymphocyte ratio as a new predictive and prognostic factor at the hearing loss of diabetic patients. Eur Arch Otorhinolaryngol, 271, 2681-6.

Walsh SR, Cook EJ, Goulder F, Justin TA, Keeling NJ (2005). Neutrophil-lymphocyte ratio as a prognostic factor in colorectal cancer. J Surg Oncol, 91, 181-4.

Yesil A, Senates E, Bayoglu IV, et al (2011). Red cell distribution width: A novel marker of activity in inflammatory bowel disease. Gut Liver, 5, 460-7. 